\title{
Axial Anomaly in SU(N) Yang-Mills Matrix Models
}

\author{
Nirmalendu Acharyya $\oplus^{1, *}$ Mahul Pandey, ${ }^{2, \dagger}$ and Sachindeo Vaidya ${ }^{3, *}$ \\ ${ }^{1}$ School of Basic Sciences, Indian Institute of Technology Bhubaneswar, Odisha 752050, India \\ ${ }^{2}$ School of Theoretical Physics, Dublin Institute for Advanced Studies, Dublin 4 D04 C932, Ireland \\ ${ }^{3}$ Centre for High Energy Physics, Indian Institute of Science, Bengaluru 560012, India
}

(Received 28 April 2021; accepted 5 August 2021; published 27 August 2021)

\begin{abstract}
The $\mathrm{SU}(N)$ Yang-Mills matrix model admits self-dual and anti-self-dual instantons. When coupled to $N_{f}$ flavors of massless quarks, the Euclidean Dirac equation in an instanton background has $n_{+}$positive and $n_{-}$negative chirality zero modes. The vacua of the gauge theory are $N$-dimensional representations of SU(2), and the (anti-) self-dual instantons tunnel between two commuting representations, the initial one composed of $r_{0}^{(1)}$ irreps and the final one with $r_{0}^{(2)}$ irreps. We show that the index $\left(n_{+}-n_{-}\right)$in such a background is equal to a new instanton charge $\mathcal{T}_{\text {new }}= \pm\left[r_{0}^{(2)}-r_{0}^{(1)}\right]$. Thus $\mathcal{T}_{\text {new }}=\left(n_{+}-n_{-}\right)$is the matrix model version of the Atiyah-Singer index theorem. Further, we show that the path integral measure is not invariant under a chiral rotation, and relate the noninvariance of the measure to the index of the Dirac operator. Axial symmetry is broken anomalously, with the residual symmetry being a finite group. For $N_{f}$ fundamental fermions, this residual symmetry is $\mathbb{Z}_{2 N_{f}}$, whereas for adjoint quarks it is $\mathbb{Z}_{4 N_{f}}$.
\end{abstract}

DOI: 10.1103/PhysRevLett.127.092002

Introduction.-A symmetry of a classical theory that cannot be implemented in its quantum counterpart is said to be broken anomalously. A well-known example of such an anomaly occurs in gauge theories with massless Dirac fermions $\Psi$. The classical action is invariant under the vector rotation $\Psi \rightarrow e^{i \alpha} \Psi$ as well as the axial rotation $\Psi \rightarrow e^{i \alpha \gamma^{5}} \Psi$, but the latter is anomalously broken [1,2]. This is the axial anomaly, which manifests in the nonconservation of the axial current $j_{A}^{\mu}=\bar{\Psi} \gamma^{\mu} \gamma^{5} \Psi$ in the quantum theory. Rather than $\partial_{\mu} j_{A}^{\mu}=0$, we have $\partial_{\mu} j_{A}^{\mu} \sim \epsilon^{\mu \nu \rho \sigma} F_{\mu \nu} F_{\rho \sigma}$, where $F_{\mu \nu}$ is the gauge curvature.

As argued by Fujikawa [3,4], the origin of the axial anomaly may be understood by examining the Euclidean path integral $\int \mathcal{D} \bar{\Psi} \mathcal{D} \Psi e^{-S_{E}^{F}}$, where $S_{E}^{F}=\int d^{4} x i \bar{\Psi} \not D \Psi$ is the fermionic action and $\not D$ is the gauge-covariant Dirac operator. Expanding $\Psi$ and $\bar{\Psi}$ in the basis of eigenfunctions of $\not D$ shows that although $S_{E}$ is invariant under axial rotations, the measure $\mathcal{D} \bar{\Psi} \mathcal{D} \Psi$ is not. The change in the measure is related to the index of the Dirac operator, which in turn is related to the instanton number $\int d^{4} x \epsilon^{\mu \nu \rho \sigma} F_{\mu \nu} F_{\rho \sigma}$ via the Atiyah-Singer index theorem.

In this Letter we will demonstrate a surprising result: the quantum $\mathrm{SU}(N)$ Yang-Mills matrix model coupled to $N_{f}$

Published by the American Physical Society under the terms of the Creative Commons Attribution 4.0 International license. Further distribution of this work must maintain attribution to the author(s) and the published article's title, journal citation, and DOI. Funded by SCOAP. massless quarks also exhibits the axial anomaly. In quantum mechanical situations, anomalies appear when the symmetry operation does not preserve the domain of the Hamiltonian [5-7]. For instance, the Hamiltonian of a free particle on a circle has a family of self-adjoint domains $\mathcal{D}_{\varphi}=\left\{f(x) \in L^{2}[-\pi, \pi]: f(x+\pi)=e^{i \varphi} f(x-\pi)\right\}$. For $\varphi \neq n \pi$, parity transformation $\mathcal{P}: x \rightarrow-x$, a classical symmetry, changes the domain $\mathcal{D}_{\varphi} \rightarrow \mathcal{D}_{-\varphi}$, resulting in the anomalous breaking of $\mathcal{P}$ [6]. Another example, where the classical scaling symmetry $S: r \rightarrow \mu r, p \rightarrow \mu^{-1} p$ is anomalously broken has been discussed in careful detail by Jackiw [7]. Anomalies have also been reported in discrete lattice formulations of Kähler-Dirac fermions [8].

Such anomalies have been shown to play an important role in the possible phases of $\theta$-QCD (quantum chromodynamics) $[9,10]$. Our demonstration of the axial anomaly closely follows the Euclidean approach: we show that there exist zero modes of the Euclidean Dirac operator in the background of (anti-) self-dual instanton gauge configurations, and the index of $\not D$ is nonzero.

In the matrix model, the instantons tunnel between the classical vacua $A_{i}=L_{i}$, where $L_{i}$ 's are the generators of $\mathrm{SU}(2)$ in any $N$-dimensional representation, with $r_{0}$ irreps (irreducible representations). The (anti-) self-dual instantons tunnelling between two distinct classical vacua say $L_{i}^{(1)}$ with $r_{0}^{(1)}$ irreps and $L_{i}^{(2)}$ with $r_{0}^{(2)}$ irreps can be associated with a new charge $\mathcal{T}_{\text {new }} \equiv\left[r_{0}^{(2)}-r_{0}^{(1)}\right]$. As we will demonstrate, the index of $\not D$ is equal to $\mathcal{T}_{\text {new }}$, giving us the matrix model version of the Atiyah-Singer index theorem. Finally, we relate the index to the noninvariance 
of the path-integral measure, which gives the anomaly. For the fermions in the fundamental representation of $\mathrm{SU}(N)$ (i.e., quarks), there remains a residual $\mathbb{Z}_{2 N_{f}}$ symmetry, while for adjoint fermions the residual symmetry is $\mathbb{Z}_{4 N_{f}}$. We emphasize that our result holds for any color $N \geq 2$.

The surprise here is that the $\mathrm{SU}(N)$ matrix model is vastly different from $\mathrm{SU}(N)$ gauge field theory. In the field theory, distinct instantons are labeled by the instanton charge $\pi_{3}[\mathrm{SU}(N)]=\mathbb{Z}$, whereas in the corresponding matrix model, they are labeled by a finite set of integers. But even though the number of distinct instantons is finite, it is enough to disturb the balance between left- and righthanded fermion zero modes, leading to the anomalous breaking of axial symmetry.

The matrix model discussed here was first presented in [11-13], and has been shown to be an excellent candidate for an effective low-energy approximation of $\mathrm{SU}(N)$ YangMills theory on $S^{3} \times \mathbb{R}$. In particular, numerical investigations of its spectrum gave excellent estimates for the low-lying glueball and hadron masses $[14,15]$. However, to serve as a correct low-energy approximation of Yang-Mills theory, this quantum-mechanical model must also exhibit the axial anomaly. Our present work provides this important conceptual support.

The matrix model-The dynamical degrees of freedom of the model are elements of $\mathcal{M}_{N}$, the set of all $3 \times\left(N^{2}-1\right)$-dimensional real matrices $M_{i a}$, or equivalently $A_{i}=M_{i a} T_{a}, T_{a}$ being the generators of $\mathrm{SU}(N)$ in the fundamental representation. Gauge transformations act on $A_{i}$ via the adjoint action $A_{i} \rightarrow h A_{i} h^{-1}, h \in \mathrm{SU}(N)$. The configuration space is the base space of principle bundle $\operatorname{AdSU}(N) \rightarrow \mathcal{M}_{N} \rightarrow \mathcal{M}_{N} / \operatorname{AdSU}(N)$. This fiber bundle being twisted [16,17], lies at the heart of the Gribov problem in Yang-Mills theory.

To describe the dynamics, we need to define a gaugecovariant time derivative of $A_{i}$. For that, we introduce a set of time-dependent real functions, conveniently named as $M_{0 a}$, and the matrix $A_{0} \equiv M_{0 a} T_{a} . A_{0}$ is the parallel transporter needed to define the covariant derivative along the temporal direction and under a gauge transformation $h(t) \in \mathrm{SU}(N), \quad A_{0} \rightarrow A_{0}^{\prime}=h A_{0} h^{-1}-\dot{h} h^{-1}$. Then, the gauge-covariant time derivative of $A_{i}$ is defined as

$$
D_{t} A_{i}=\dot{A}_{i}-i\left[A_{0}, A_{i}\right]
$$

which transforms as $D_{t} A_{i} \rightarrow h\left(D_{t} A_{i}\right) h^{-1}$.

The curvature $F_{i j}$ is obtained by the pullback of the Maurer-Cartan equation of $\mathrm{SU}(N)$ to $S^{3}: F_{i j}=-\epsilon_{i j k} R^{-1} A_{k}-$ $i\left[A_{i}, A_{j}\right]$, where $R$ is the radius of the $S^{3}$ (for a derivation of the matrix model, see the Supplemental Material [18]). The chromoelectric field $E_{i} \equiv D_{t} A_{i}$ and the chromomagnetic field $B_{i} \equiv \frac{1}{2} \epsilon_{i j k} F_{j k}$ are given by
$E_{i}=\dot{A}_{i}-i\left[A_{0}, A_{i}\right], \quad B_{i}=-\frac{1}{R} A_{i}-\frac{i}{2} \epsilon_{i j k}\left[A_{j}, A_{k}\right]$.

The Yang-Mills Lagrangian is

$$
L_{\mathrm{YM}}=\frac{R^{3}}{g^{2}}\left[\operatorname{Tr}\left(D_{t} A_{i}\right)^{2}-V(A)\right], \quad V(A) \equiv \operatorname{Tr} B_{i} B_{i} .
$$

The quarks $\Psi$ are Grassmann-valued matrices that depend only on time, and transform in the fundamental representation of $\mathrm{SU}(N)$ and in the spin- $\frac{1}{2}$ representation of spatial rotations. The Lagrangian with minimally coupled massless quarks is $L=L_{\mathrm{YM}}+L_{F}$ where $L_{F}=$ $R^{3} \bar{\Psi}\left[i \gamma^{0} D_{t}+\gamma^{i} A_{i}-(3 / 2 R) \gamma^{5} \gamma^{0}\right] \Psi$ is the gauge-covariant Dirac Lagrangian $[13,19]$

It is convenient to rescale to dimensionless variables $A_{\mu} \rightarrow R A_{\mu}, \Psi \rightarrow R^{3 / 2} \Psi$, and $t \rightarrow R^{-1} t$. Then performing a Wick rotation $t \rightarrow-i \tau$ and $A_{0} \rightarrow i A_{0}$ in $L$ gives us the Euclidean action $S_{E}=S_{E}^{\mathrm{YM}}+S_{E}^{F}$ [20] with

$$
S_{E}^{\mathrm{YM}}=\frac{1}{g^{2} R} \int d \tau\left[\operatorname{Tr} \mathbb{E}_{i} \mathbb{E}_{i}+V_{0}\right], \quad S_{E}^{F}=\frac{1}{R} \int d \tau \Psi^{\dagger} i \not D \Psi,
$$

where $\mathbb{E}_{i}\left(\equiv \mathbb{E}_{i}^{a} T_{a}\right)=\left(\partial A_{i} / \partial \tau\right)-\left[A_{0}, A_{i}\right]$ is the Euclidean chromoelectric field, and the potential $V_{0}=\left(A_{i}+\right.$ $\left.(i / 2) \epsilon_{i j k}\left[A_{j}, A_{k}\right]\right)^{2}$ is obtained by setting $R=1$ in (3). $\not D$ is the Euclidean Dirac operator, $i \not D=(\partial / \partial \tau)-A_{0}-$ $\gamma^{0} \gamma^{i} A_{i}-\frac{3}{2} \gamma^{5}$. As, the $R$ and $g$ dependence of $S_{E}^{\mathrm{YM}}$ and $S_{E}^{F}$ is just via multiplicative prefactors, we choose $R=1$ and $g=1$ without any loss of generality.

The classical action $S_{E}$ is indeed invariant under the axial rotation $\Psi \rightarrow e^{i \alpha \gamma^{5}} \Psi$ and has $U(1)_{A}$ symmetry. However as we demonstrate below, the quantum effects do not preserve this symmetry.

Classical vacuum configurations of Eq. (3) are given by those $A_{i}$ which satisfy $V(A)=0$, i.e., $\left[A_{i}, A_{j}\right]=i \epsilon_{i j k} A_{k}$. This has solutions $A_{i}=L_{i}$, where $L_{i}$ are generators of the Lie algebra of SU(2). There are multiple degenerate vacua which correspond to the matrices $A_{i}$ forming a general $N$ dimensional representation of $L_{i}$.

The tunnelling between degenerate classical minima is captured by instantons, the finite action solutions of equations of motion of $S_{E}^{\mathrm{YM}}$ in Eq. (4). This action is extremized when $\mathbb{E}_{i}= \pm B_{i}$, whose solutions with a plus (minus) sign give the self-dual (anti-self-dual) instantons.

Using the gauge freedom, the self-dual equation can be transformed to the temporal gauge $A_{0}=0$ to read

$$
\frac{d A_{i}}{d \tau}= \pm\left(-A_{i}-\frac{i}{2} \epsilon_{i j k}\left[A_{j}, A_{k}\right]\right)
$$

Substituting the ansatz 


$$
A_{i}=\phi(\tau) L_{i}^{(1)}+[1-\phi(\tau)] L_{i}^{(2)}, \quad\left[L_{i}^{(1)}, L_{j}^{(2)}\right]=0
$$

into Eq. (5), we obtain

$$
\frac{\partial \phi_{s}}{\partial \tau}=-\phi_{s}\left(1-\phi_{s}\right), \quad \frac{\partial \phi_{a}}{\partial \tau}=\phi_{a}\left(1-\phi_{a}\right),
$$

which have solutions

$$
\phi_{s}(\tau)=\frac{1}{1+e^{\left(\tau-\tau_{0}\right)}}, \quad \phi_{a}(\tau)=\frac{1}{1+e^{-\left(\tau-\tau_{0}\right)}} .
$$

Here, $s$ denotes the self-dual instanton, and $a$ denotes the anti-self-dual instanton. If $\left[L_{i}^{(1)}, L_{j}^{(2)}\right] \neq 0$, we would have to solve the second order equation of motion instead of the simpler first order Eq. (5). In general, $L_{i}^{(\alpha)}$ is a direct sum of $r_{0}^{(\alpha)}$ irreps with each irrep $L_{i}^{(\alpha), r}$ with $j_{r}^{(\alpha)}=\left[\left(N_{r}^{(\alpha)}-1\right) / 2\right]$.

If we gauge transform the solutions by $h(\tau)$ to obtain a nonzero $A_{0}$, the transformed instanton solutions go between $L_{i}^{(1,2)}$ and $h_{b} L_{i}^{(2,1)} h_{b}^{-1}$, where $h_{b}=h(-\infty)$ for the self-dual and $h_{b}=h(\infty)$ for the anti-self-dual instanton. This does not affect any of the subsequent arguments, since we focus only on gauge-invariant quantities. These instanton solutions have been studied earlier by $[21,22]$ in a different context.

The instanton number $\mathcal{T}=c \int_{-\infty}^{\infty} d \tau \operatorname{Tr} \mathbb{E}_{i} B_{i}$ is the integral of a total derivative. The normalization factor $c$ is fixed to 4 so that $\mathcal{T}$ is an integer. For the (anti-) self-dual instantons

$\mathcal{T}_{s}=-\mathcal{T}_{a}=\frac{2}{3} \sum_{\alpha=1}^{2} \epsilon_{\alpha \beta} \sum_{r=1}^{r_{0}^{(\alpha)}} j_{r}^{(\alpha)}\left[j_{r}^{(\alpha)}+1\right]\left[2 j_{r}^{(\alpha)}+1\right]$.

However for the (anti-)self-dual instanton, we can construct another charge that is expressible only in terms of $r_{0}^{(\alpha)}$, the number of irreps at $\tau= \pm \infty$. Defining $\mathbb{E}_{i}^{\prime} \equiv$ $\left(d \phi_{s / a} / d \tau\right)\left[e_{i}^{(2)}-e_{i}^{(1)}\right]$ with

$e_{i}^{(\alpha)}=\bigoplus_{r=1}^{r_{0}^{(\alpha)}} e_{i}^{(\alpha), r}, \quad e_{i}^{(\alpha), r}=\frac{3}{\left[j_{r}^{(\alpha)}+1\right]\left[2 j_{r}^{(\alpha)}+1\right]} L_{i}^{(\alpha), r}$,

the new charge is given by

$$
\mathcal{T}_{\text {new }}=4 \int d \tau \operatorname{Tr} \mathbb{E}_{i}^{\prime} B_{i}= \pm\left[r_{0}^{(2)}-r_{0}^{(1)}\right] .
$$

$\mathcal{T}_{\text {new }}$ is still an integral of a total $\tau$ derivative and depends only on $\left[r_{0}^{(2)}-r_{0}^{(1)}\right]$, rather than the Casimirs or other labels of each individual irrep. As we will demonstrate below,
$\mathcal{T}_{\text {new }}$ for a (anti-)self-dual instanton is equal to the index of the Dirac operator.

Index of $\not D$.-The Dirac operator obeys $\left\{\not D, \gamma^{5}\right\}=0$ and consequently, for every eigenfunction $\psi_{n}$ of $\not D$ with a nonzero eigenvalue $\lambda_{n}$, there is an eigenfunction $\gamma^{5} \psi_{n}$ with eigenvalue $\left(-\lambda_{n}\right)$. The eigenfunctions with zero eigenvalue (the zero modes) can also be arranged to be eigenfunctions of $\gamma^{5}$ :

$$
\not \supset \chi_{k}^{ \pm}=0, \quad \gamma^{5} \chi_{k}^{ \pm}= \pm \chi_{k}^{ \pm}, \quad k=1,2, \ldots, n_{ \pm} .
$$

but $n_{+}$and $n_{-}$need not be equal. The index of $\not D$ is defined as ind $\not D=n_{+}-n_{-}$.

In the Weyl basis, the Dirac operator is

$\not D=\left(\begin{array}{cc}0 & \mathcal{L} \\ \mathcal{L}^{\dagger} & 0\end{array}\right), \quad \mathcal{L} \equiv-i\left(\frac{d}{d \tau}-A_{0}+\sigma_{i} A_{i}+\frac{3}{2}\right)$.

We solve for the zero modes $\Psi_{\alpha A}$ of $\not D$ in the temporal gauge. If $A_{0} \neq 0$, the corresponding zero mode is obtained via a gauge transformation on $\Psi$. In any case, $n_{ \pm}$and the index do not change under the gauge transformation, and we can make our entire argument in temporal gauge.

For the (anti-) self-dual instanton, $\sigma_{i} A_{i}(\tau)+3 / 2$ is a $2 N$-dimensional Hermitian matrix whose eigenvalues $\xi_{i}(\tau)$ are time dependent, but the eigenvectors are not. So $\mathcal{L}$ can be diagonalized and $\not D$ brought to the form

$$
\not D=\left(\begin{array}{cc}
0 & \mathcal{L}_{d} \\
\mathcal{L}_{d}^{\dagger} & 0
\end{array}\right), \quad \mathcal{L}_{d}=-i\left(\frac{d}{d \tau}+\Sigma\right),
$$

where $\Sigma=\operatorname{diag}\left[\xi_{1}(\tau), \xi_{2}(\tau), \ldots, \xi_{2 N}(\tau)\right]$. In the new basis, the positive and negative chirality zero modes are of the form $\chi^{+}=\left(0, \psi^{+}\right)^{T}$ and $\chi^{-}=\left(\psi^{-}, 0\right)^{T}$, respectively, where the $\psi^{ \pm}$satisfy $\mathcal{L}_{d} \psi^{+}=0$ and $\mathcal{L}_{d}^{\dagger} \psi^{-}=0$. The index is given by ind $\not D=\operatorname{dim} \operatorname{Ker}\left(\mathcal{L}_{d}\right)-\operatorname{dim} \operatorname{Ker}\left(\mathcal{L}_{d}^{\dagger}\right)$.

For the Dirac operator [Eq. (14)], there is a neat way to determine the index from $\xi_{i}(\tau \rightarrow \pm \infty)$ using Callias' index theorem [23]:

$$
\operatorname{ind} \not D=\frac{1}{2} \sum_{i=1}^{2 N}\left[\operatorname{sgn} \xi_{i}(\tau \rightarrow-\infty)-\operatorname{sgn} \xi_{i}(\tau \rightarrow \infty)\right] .
$$

Let us apply Eq. (15) to the simple case when $A_{i}=\phi_{s / a}(\tau) L_{i}$, i.e., when $L_{i}^{(2)}=0$ and $L_{i}^{(1)}=L_{i}$. Say $L_{i}$ consists of $r_{0}$ irreducible blocks with each block of dimension $N_{r} \equiv\left(2 j_{r}+1\right)$. In each block, $\sigma_{i} A_{i}+3 / 2$ is a $2 N_{r}$-dimensional matrix and has $\left(N_{r}-1\right)$ degenerate eigenvalues $\lambda_{1, s / a} \equiv \frac{1}{2}\left[3-\phi_{s / a}(\tau)\left(N_{r}+1\right)\right]$, and $\left(N_{r}+1\right)$ degenerate eigenvalues $\lambda_{2, s / a} \equiv \frac{1}{2}\left[3+\phi_{s / a}(\tau)\left(N_{r}-1\right)\right]$. As $\lambda_{2, s / a}>0$ has same sign at both $\tau=-\infty$ and $\tau=\infty$, their contribution in Eq. (15) vanishes, and consequently the index in (anti-) self-dual background is 


$$
\operatorname{ind} \not D_{s}=\frac{1}{2} \sum_{r=1}^{r_{0}}\left(N_{r}-1\right)\left[1-\frac{2-N_{r}}{\left|2-N_{r}\right|}\right]=-\operatorname{ind} \not D_{a} .
$$

For each block with $N_{r}>2$, the term in the square brackets above evaluates to 2 . There exist $N_{r}-1$ normalizable zero modes of $\not D$ with one chirality $(+1$ for the self-dual instanton background and -1 for anti-self-dual one) and none with the opposite chirality. For blocks with $N_{r}=1$, the index evaluates to 0 and consequently there are no normalizable zero modes.

For the blocks with $N_{r}=2$, it seems at first sight that the index [Eq. (16)] is undefined. For this case, one eigenvalue of $\Sigma$ vanishes at either $\tau=-\infty$ or $+\infty$, depending on whether the background is self-dual or anti-self-dual. Thus for large $|\tau|$, the Dirac operator resembles that of a free particle. The corresponding zero mode is not strictly normalizable but is delta-function normalizable: it is a zero energy resonance, or a threshold state.

We show the normalizable and the non-normalizable zero modes for $N_{r}=2$ and $N_{r}=3$ in Fig. 1 (explicit form of the solutions $\psi_{s / a, n}^{ \pm}$is given in the Supplemental Material [18]).

As we shall see below, both zero modes and zero energy resonance states have a nonzero contribution to the axial anomaly. To correctly take into account all contributions, one must extend the definition of ind $\not D$ to include zero modes as well as zero energy resonances with positive and negative chirality [24].

In general for the ansatz [Eq. (6)], we find that ind $\not_{s}=$ $-\operatorname{ind} \not D_{a}=r_{0}^{(2)}-r_{0}^{(1)}$, where $r_{0}^{(\alpha)}$ is the number of irreps in $L_{i}^{(a)}$. Thus the index is equal to $\mathcal{T}_{\text {new }}$ defined in Eq. (11). This yields the matrix model version of the Atiyah-Singer index theorem

$$
\operatorname{ind} \not D=\mathcal{T}_{\text {new }} \text {. }
$$

We emphasize that $\mathcal{T}_{\text {new }}$ is a quantity computed from the pure gauge sector, while ind $\not D$ counts the difference between the number of fermion zero modes of opposite chiralities. It is remarkable that there exists such a simple relation between the two: a priori, there is no reason to expect this equality. Moreover, as we will show further below, this relation between the charge and the index can be suitably adapted for adjoint fermions as well, hinting toward its universal nature in the context of the matrix model. Despite the demonstration of this equality, we are not aware of an explanation of its geometric or topological origin.

Noninvariance of fermion measure.-We adapt Fujikawa's method to demonstrate the axial anomaly and its relation to ind $\not D$. Specifically, we show that under a $U(1)_{A}$ transformation, the measure of the fermionic path integral is not invariant. The Jacobian of the transformation gives the integrated anomaly.

In the fermionic path integral $\int \mathcal{D} \bar{\Psi} \mathcal{D} \Psi e^{-S_{E}^{F}}$, we expand the fermionic variables $\Psi=\sum a_{n} \Phi_{n}$ and $\bar{\Psi}=\sum_{n} b_{n} \Phi_{n}$, where $\Phi_{n}$ 's are eigenfunctions of $\not D$ with nonzero eigenvalues, zero modes as well as zero-energy resonances. In this basis, the fermionic path integral measure is given by $\quad d \mu \equiv \mathcal{D} \bar{\Psi} \mathcal{D} \Psi=\prod_{n, m} d a_{n} d b_{m} . \quad$ Under $\quad$ a $U(1)_{A}$ rotation with an angle $\alpha$, the transformed variables $\Psi^{\prime}$ can be expanded as $\Psi^{\prime}=\sum a_{n}^{\prime} \Phi_{n}, \bar{\Psi}^{\prime}=\sum_{n} b_{n}^{\prime} \Phi_{n}$ where the coefficients transform linearly: $a_{n}^{\prime}=C_{m n} a_{m}$ and $b_{n}^{\prime}=C_{m n} b_{m}$.

Consequently $d \mu$ transforms as $d \mu \rightarrow[\operatorname{det} C]^{-2} d \mu$. The Jacobian is $[\operatorname{det} C]^{-1}=e^{-i \int d \tau \alpha \mathcal{A}(\tau)}$, where the anomaly function $\mathcal{A}(\tau)$ is defined as $\mathcal{A}(\tau)=\sum_{n} \Phi_{n}^{\dagger} \gamma^{5} \Phi_{n}$. In $\mathcal{A}(\tau)$, the summation is over an infinite number of modes and hence divergent. We introduce a gauge-invariant regulator $e^{-\beta \not D^{2}}$ and formally take the limit $\beta \rightarrow 0$ at the end: $\mathcal{A}(\tau)=\lim _{\beta \rightarrow 0} \sum_{n} \Phi_{n}^{\dagger} \gamma^{5} e^{-\beta \not \not^{2}} \Phi_{n}$.

We already saw that for nonzero eigenvalues, the eigenfunctions $\Phi_{n}$ and $\gamma^{5} \Phi_{n}$ of $\not D$ are orthogonal, and hence do not contribute to the summation. However, the zero modes and the zero-energy scattering states can have nonzero contribution to $\mathcal{A}(\tau)$.

Setting $\alpha=$ constant, the integrated anomaly function is

$$
\int d \tau \mathcal{A}(\tau)=\int d \tau\left[\sum_{k=1}^{n_{+}} \chi_{k}^{+\dagger} \gamma^{5} \chi_{k}^{+}+\sum_{k=1}^{n_{-}} \chi_{k}^{-\dagger} \gamma^{5} \chi_{k}^{-}\right]
$$

Using Eq. (12), we get $\int d \tau \mathcal{A}(\tau)=\left(n_{+}-n_{-}\right)=\operatorname{ind} \not D$.

Thus in a background gauge configuration where the Dirac operator has a nonzero index, the fermion measure is
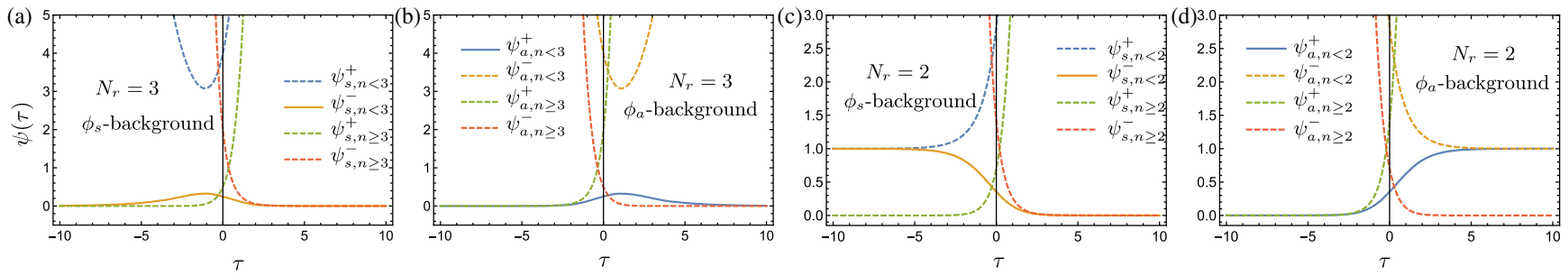

FIG. 1. The solutions of $\not \psi_{s / a, n}^{ \pm}=0$ with (a) $N_{r}=3$ in self-dual background, (b) $N_{r}=3$ in anti-self-dual background, (c) $N_{r}=2$ in self-dual background, (d) $N_{r}=2$ in anti-self-dual background. 
not invariant under axial transformations, and axial symmetry is anomalously broken.

Because ind $\not D$ is always an integer, $e^{\mp 2 i \alpha \int d \tau \mathcal{A}(\tau)}=1$ when $\alpha=n \pi(n \in \mathbb{Z})$ for any $N$. For axial rotations with $\alpha=n \pi$, the anomaly vanishes. Thus the $U(1)_{A}$ is anomalously broken to $\mathbb{Z}_{2}$, the residual axial symmetry.

For $N_{f}$ flavors, the Dirac operator is diagonal in flavor. Its spectrum is $N_{f}$ copies of the spectrum of $\not D$ for a single flavor. The axial symmetry is now broken to $\mathbb{Z}_{2 N_{f}}$.

Adjoint Weyl Fermion.-For supersymmetric gauge matrix models, the adjoint Weyl fermions $\lambda$ are of relevance $[25,26]$. We show that axial symmetry is anomalously broken in this case too.

The Euclidean fermionic action can be written as

$$
S_{E}^{F}=\int d t \lambda^{\dagger}\left(\partial_{\tau}-\mathcal{F}_{0}+\sigma^{i} \mathcal{F}_{i}+\frac{3}{2}\right) \lambda
$$

where $\mathcal{F}_{0}=M_{0 a} G_{a}, \mathcal{F}_{i}=M_{i a} G_{a}$, and $G_{a}=-i f_{a b c}$ are the $\mathrm{SU}(N)$ generators in the adjoint representation. As before, we choose the temporal gauge.

For the ansatz [Eq. (6)], $\mathcal{F}_{i}$ takes the form $\mathcal{F}_{i}=$ $\phi_{s / a} \mathcal{J}_{i}^{(1)}+\left(1-\phi_{s / a}\right) \mathcal{J}_{i}^{(2)}$ where $\mathcal{J}_{i}^{(\alpha)}$ are the representations of $\mathrm{SU}(2)$ obtained by embedding $L_{i}^{(\alpha)}$ in $\left(N^{2}-1\right)$ dimensions. It is straightforward to show that $\mathcal{J}_{i}$ are given by one singlet representation removed from the following direct sum

$$
\begin{aligned}
& \bigoplus_{r, q}\left[\left(N_{r}+N_{q}-1\right) \oplus\left(N_{r}+N_{q}-3\right) \oplus \cdots\right. \\
& \left.\oplus\left(\left|N_{r}-N_{q}\right|+1\right)\right] .
\end{aligned}
$$

Thus $\mathcal{J}_{i}^{(\alpha)}$ is a direct sum of $q_{0}^{(\alpha)}$ irreps $\mathcal{J}_{i}^{(\alpha), q}$ of dimension $\mathcal{N}_{q}^{(\alpha)}$, with $\sum_{q=1}^{q_{0}^{(\alpha)}} \mathcal{N}_{q}^{(\alpha)}=N^{2}-1$. Just as before, we define $\quad \mathcal{E}_{i}^{\prime}=\left(d \phi_{s / a} / d \tau\right)\left[e_{i}^{(2)}-e_{i}^{(1)}\right] \quad$ and $\quad \mathcal{B}_{i}=-\mathcal{F}_{i}-$ $(i / 2) \epsilon_{i j k}\left[\mathcal{F}_{j}, \mathcal{F}_{k}\right]$ where $e_{i}^{(\alpha)}=\bigoplus_{q=1}^{q_{0}^{(\alpha)}} e_{i}^{(\alpha), q}$ [the $e_{i}^{(\alpha), q}$ are as in Eq. (10)]. The instanton charge for the embedding is given by

$$
\mathcal{T}_{\text {new }}^{\text {adj }}=\int d \tau \operatorname{Tr} \mathcal{E}_{i}^{\prime} \mathcal{B}_{i}= \pm\left[q_{0}^{(2)}-q_{0}^{(1)}\right] .
$$

The index calculation is exactly as in Eqs. (15) and (16), by replacing $N_{r}^{(\alpha)}$ with $\mathcal{N}_{q}^{(\alpha)}$. By correctly taking into account the zero modes as well as the zero-energy resonances, the index is now given by ind $\not D=\mathcal{T}_{\text {new }}^{\text {adj }}$.

In the adjoint case, all eigenvalues of $\not D$ are doubly degenerate. For every eigenvector $\varphi_{n}$ of $\not D$ with eigenvalue $\lambda_{n}$, there exists an independent eigenvector $\left(\sigma_{2} \otimes \mathbf{1}\right) \varphi^{*}$ with the same eigenvalue. Consequently, the index is always an even integer, and so $e^{-2 i \alpha \text { ind } \not}=1$ when $\alpha=(n \pi / 2), \quad n \in \mathbb{Z}$. Therefore a single adjoint Weyl fermion breaks the $U(1)_{A}$ axial symmetry to a residual $\mathbb{Z}_{4}$ subgroup. For $N_{f}$ flavors, the residual symmetry is $\mathbb{Z}_{4 N_{f}}$.

Discussion.-Though the Yang-Mills matrix model is very different from the corresponding field theory, it nevertheless retains important nonperturbative features of the field theory. As we have demonstrated here, the axial anomaly is one such feature. In the usual discussion of the axial anomaly in gauge field theories, only the irreducible connections are considered, and it is the instanton number of such connections that is related to the fermion zero modes. A priori, there is no reason that the residual axial symmetry in the matrix model should match the corresponding field theory result, and it is surprising that it matches for the case of fundamental fermions. Whether this coincidence has a deeper significance requires further investigation.

Our result on the anomaly provides a strong conceptual support for the numerical investigations of the matrix model [15]. In addition to reproducing the masses of light hadrons with surprising accuracy, the numerics also show that the pseudoscalar mesons are much lighter than their scalar counterparts. Furthermore, it also finds the $\eta^{\prime}$ meson to be considerably heavier than the $\eta$ meson. The result on the axial anomaly presented here serves to strengthen the position of the $\mathrm{SU}(3)$ gauge matrix model as an effective low-energy approximation of QCD.

The axial anomaly is present for any $\mathrm{SU}(N)$ gauge group, and there is no reason to expect that it is washed out in the large $N$ limit.

There is a plethora of matrix models inspired by [27-29] that have been studied in the literature and which remain a subject of continuing interest [25,30-34]. These models have a non-Abelian gauge symmetry and fermions, and in light of the results presented here, the implications of the axial anomaly for these models need to be understood. Similarly, whether this axial anomaly has a role to play in quantum systems with emergent non-Abelian gauge symmetry like the multimodal Rabi model [35] is an interesting and open question.

We thank Denjoe O'Connor and V. Parameswaran Nair for discussions, and suggesting the use of Callias' index theorem.

*nirmalendu@iitbbs.ac.in

†mpandey@stp.dias.ie

*vaidya@cts.iisc.ac.in

[1] S. L. Adler, Phys. Rev. 177, 2426 (1969).

[2] J. S. Bell and R. Jackiw, Nuovo Cimento A 60, 47 (1969).

[3] K. Fujikawa, Phys. Rev. Lett. 42, 1195 (1979).

[4] K. Fujikawa, Phys. Rev. D 21, 2848 (1980); 22, 1499(E) (1980). 
[5] S. Elitzur, Y. Frishman, E. Rabinovici, and A. Schwimmer, Nucl. Phys. B273, 93 (1986).

[6] J. G. Esteve, Phys. Rev. D 34, 674 (1986).

[7] R. Jackiw, M. A. B. Bg Memorial Volume, edited by A. Ali and P. Hoodbhoy (World Scientific, Singapore, 1991), pp. $25-42$.

[8] S. Catterall, J. Laiho, and J. Unmuth-Yockey, J. High Energy Phys. 10 (2018) 013.

[9] D. Gaiotto, A. Kapustin, Z. Komargodski, and N. Seiberg, J. High Energy Phys. 05 (2017) 091.

[10] Y. Kikuchi and Y. Tanizaki, Prog. Theor. Exp. Phys. (2017), $113 \mathrm{~B} 05$.

[11] A. P. Balachandran, S. Vaidya, and A. R. de Queiroz, Mod. Phys. Lett. A 30, 1550080 (2015).

[12] A. P. Balachandran, A. de Queiroz, and S. Vaidya, Int. J. Mod. Phys. A 30, 1550064 (2015).

[13] M. Pandey and S. Vaidya, J. Math. Phys. (N.Y.) 58, 022103 (2017).

[14] N. Acharyya, A. P. Balachandran, M. Pandey, S. Sanyal, and S. Vaidya, Int. J. Mod. Phys. A 33, 1850073 (2018).

[15] M. Pandey and S. Vaidya, Phys. Rev. D 101, 114020 (2020).

[16] I. M. Singer, Commun. Math. Phys. 60, 7 (1978).

[17] M. S. Narasimhan and T. R. Ramadas, Commun. Math. Phys. 67, 121 (1979).

[18] See Supplemental Material at http://link.aps.org/ supplemental/10.1103/PhysRevLett.127.092002 for the construction of the matrix model and the explicit zero modes of the Dirac operator.

[19] D. Sen, J. Math. Phys. (N.Y.) 27, 472 (1986).
[20] D. J. Gross, R. D. Pisarski, and L. G. Yaffe, Rev. Mod. Phys. 53, 43 (1981).

[21] P. B. Kronheimer, J. Diff. Geom. 32, 473 (1990).

[22] C. Bachas, J. Hoppe, and B. Pioline, J. High Energy Phys. 07 (2001) 041.

[23] C. Callias, Commun. Math. Phys. 62, 213 (1978).

[24] D. Bolle, F. Gesztesy, H. Grosse, W. Schweiger, and B. Simon, J. Math. Phys. (N.Y.) 28, 1512 (1987).

[25] C. T. Asplund, F. Denef, and E. Dzienkowski, J. High Energy Phys. 01 (2016) 055.

[26] V.E. Díez, M. Pandey, and S. Vaidya, Phys. Rev. D 102, 074024 (2020).

[27] N. Ishibashi, H. Kawai, Y. Kitazawa, and A. Tsuchiya, Nucl. Phys. B498, 467 (1997).

[28] T. Banks, W. Fischler, S. H. Shenker, and L. Susskind, Phys. Rev. D 55, 5112 (1997).

[29] D. E. Berenstein, J. M. Maldacena, and H. S. Nastase, J. High Energy Phys. 04 (2002) 013.

[30] V. G. Filev and D. O'Connor, J. High Energy Phys. 05 (2016) 167.

[31] Y. Asano, V. G. Filev, S. Kováčik, and D. O’Connor, J. High Energy Phys. 07 (2018) 152.

[32] S. Hadizadeh, B. Ramadanovic, G. W. Semenoff, and D. Young, Phys. Rev. D 71, 065016 (2005).

[33] M. S. Costa, L. Greenspan, J. Penedones, and J. Santos, J. High Energy Phys. 03 (2015) 069.

[34] X. Han and S. A. Hartnoll, Phys. Rev. X 10, 011069 (2020).

[35] J. Larson and S. Levin, Phys. Rev. Lett. 103, 013602 (2009). 